\title{
Hyperoside inhibits biofilm formation of Pseudomonas aeruginosa
}

\author{
YIXUAN SUN, FENGJUN SUN, WEI FENG, XUEWEN QIU, YAO LIU, \\ BO YANG, YONGCHUAN CHEN and PEIYUAN XIA
}

\begin{abstract}
Department of Pharmacy, Southwest Hospital, Third Military Medical University, Chongqing 400038, P.R. China
\end{abstract}
Received September 8, 2015; Accepted October 21, 2016

DOI: 10.3892/etm.2017.4641

\begin{abstract}
Pseudomonas aeruginosa (P. aeruginosa) is a common pathogen in hospital-acquired infection and is readily able to form biofilms. Due to its high antibiotic resistance, traditional antibacterial treatments exert a limited effect on $P$. aeruginosa biofilm infections. It has been indicated that hyperoside inhibits $P$. aeruginosa PAO1 (PAO1) biofilm formation without affecting growth. Therefore, the current study examined the biofilm formation and quorum sensing (QS) system of PAO1 in the presence of hyperoside. Confocal laser scanning microscopy analysis demonstrated that hyperoside significantly inhibited biofilm formation. It was also observed that hyperoside inhibited twitching motility in addition to adhesion. Data from reverse transcription-quantitative polymerase chain reaction indicated that hyperoside inhibited the expression of lasR, lasI, rhlR and rhlI genes. These results suggest that the QS-inhibiting effect of hyperoside may lead to a reduction in biofilm formation. However, the precise mechanism of hyperoside on P. aeruginosa pathogenicity remains unclear and requires elucidation in additional studies.
\end{abstract}

\section{Introduction}

Pseudomonas aeruginosa ( $P$. aeruginosa) is a common pathogen in hospital-acquired infections (1). Due to increasing multidrug resistance, $P$. aeruginosa infection is an increasingly common cause of mortality and morbidity (2). The mechanisms of antibiotic resistance in P. aeruginosa include the expression of multiple antibiotic modifying enzymes, antibiotic efflux pumps and acquisition of chromosomally or plasmid encoded antibiotic resistance genes. Additionally, chromosomal mutations and lower membrane permeability for the antibiotics also contribute to antibiotic resistance (3).

Correspondence to: Professor Peiyuan Xia, Department of Pharmacy, Southwest Hospital, Third Military Medical University, 30 Gaotanyan Street, Chongqing 400038, P.R. China

E-mail: peiyuan_xia@163.com

Key words: hyperoside, Pseudomonas aeruginosa, biofilm, quorum sensing, twitching motility, adhesion
P. aeruginosa is a biofilm-forming pathogen and is difficult to eradicate due to its high antibiotic resistance and the ability of the biofilm to evade the immune system (4-7). Quorum sensing (QS) is a system of stimuli and response correlated to population density. P. aeruginosa uses the QS system to coordinate gene expression according to the density of its local population. Thus, it can coordinate certain behaviors such as biofilm formation, virulence and antibiotic resistance. QS inhibitors (QSIs) are the most well reported alternative therapeutics that can be used to overcome the problem of increasing antibiotic resistance in $P$. aeruginosa. QSIs target the virulence of the organism and therefore are also termed antipathogenic drugs. The virulence of P. aeruginosa depends on its cell-to-cell communication system, or QS system that uses diffusible signaling molecules that accumulate with increasing cell density and allows $P$. aeruginosa to trigger coordinated responses and achieve outcomes that would otherwise remain impossible to achieve by individual bacterium (8). Previous studies have demonstrated that traditional treatments for bacteria exert some effect on biofilm infections $(9,10)$. Therefore, the effects of constituents from marine organisms, traditional Chinese herbs and plants (11-13) on biofilm infections are being assessed.

Flavonoids are plant polyphenols present in vegetables, fruits and beverages of plant origin and are well known for their antipyretic, analgesic and anti-inflammatory physiological properties (14-18). Hyperoside is a type of modified flavonoid. It has been demonstrated that hyperoside has weak antibacterial activity against gram-positive bacteria and no antibacterial activity against gram-negative bacteria $(19,20)$. Furthermore, it has been identified that hyperoside exhibits an inhibitory effect on $P$. aeruginosa PAO1 (PAO1) biofilms (21). While the incidence of infections caused by antibiotic resistant strains has increased, the discovery of novel classes of antibiotics has slowed down which made it imperative to search for alternative treatment strategies. Therefore, the current study examined the biofilm formation, adhesion and motility of PAO1 in the presence of hyperoside. And the hyperoside may become a more effective method to treat the infection of $P$. aeruginosa.

\section{Materials and methods}

Bacterial strains and culture conditions. P. aeruginosa PAO1 was acquired from Bioplus Biotech Co., Ltd. (Shanghai, China) and cultured in Lubria-Bertani (LB) medium (BD 
Diagnostics, Sparks Glencoe, MD, USA) at $37^{\circ} \mathrm{C}$ for $24 \mathrm{~h}$ in all experiments. The hyperoside was purchased from Dalian Meilun Biotechnology Co., Ltd. (Dalian, China).

Dose effect of hyperoside on biofilm formation. PAO1 was activated in LB medium overnight at $37^{\circ} \mathrm{C}$ prior to $1: 1,000$ $(\mathrm{v} / \mathrm{v})$ dilution in a tissue culture microtiter plate. The final concentrations of hyperoside in the tissue culture microtiter plate were $8,16,32,64,128$ and $256 \mu \mathrm{g} / \mathrm{ml}$. Following $24 \mathrm{~h}$ incubation at $37^{\circ} \mathrm{C}$, the medium was removed and wells were washed three times with $\mathrm{ddH}_{2} \mathrm{O}$. The microtiter plate was dried prior to the addition of $1 \%$ crystal violet (Sigma-Aldrich; Merck Millipore, Darmstadt, Germany) for $15 \mathrm{~min}$ at room temperature. Following staining, the dye was removed and the wells were washed three times with tap water. The microtiter plate was dried prior to the addition of $30 \%$ glacial acetic acid to solubilize the dye bound to the biofilm. The absorbance was measured by xMark Microplate Spectrophotometer (Bio-Rad, Laboratories, Inc., Hercules, CA, USA) at $590 \mathrm{~nm}$. The optimal concentration for biofilm inhibition was used in the later experiments.

Growth assays. Cells were grown in LB medium in the presence or absence of $16 \mu \mathrm{g} / \mathrm{ml}$ hyperoside. Bacterial culture turbidity was measured by xMark Microplate Spectrophotometer (Bio-Rad, Laboratories, Inc) at $600 \mathrm{~nm}$ at intervals of $0 \mathrm{~h}$ up to $24 \mathrm{~h}$.

Microscopy analysis. Confocal laser scanning microscopy (CLSM) was performed to analyze the effect of hyperoside on the PAO1 biofilm at $24 \mathrm{~h}$. Prior to the CLSM experiments, cell cultures were divided into control and hyperoside $(16 \mu \mathrm{g} / \mathrm{ml})$-treated groups. Biofilms on the culture dish were fixed with $2.5 \%$ glutaraldehyde at room temperature for $3 \mathrm{~h}$. Following washing with phosphate-buffered saline (PBS), $5 \mu \mathrm{g} / \mathrm{ml}$ propidium iodide (Sigma-Aldrich) was added and the biofilms were incubated for $15 \mathrm{~min}$ at $4^{\circ} \mathrm{C}$. The plate was then washed, $50 \mu \mathrm{g} / \mathrm{ml}$ fluorescein isothiocyanate-concanavalin A (Sigma-Aldrich) was added and the plate was incubated for $30 \mathrm{~min}$ at $4^{\circ} \mathrm{C}$. The stained biofilm was then observed using CLSM.

Adhesion assays. Adhesion assays were performed as previously described with minor modifications (22). Following PAO1 activation, the control and hyperoside groups were cultured in a 96-well microtiter plate and incubated for $4 \mathrm{~h}$ at $37^{\circ} \mathrm{C}$. Following incubation, the attached cells were stained with filtered $1 \%$ crystal violet (Sigma-Aldrich) at room temperature for $15 \mathrm{~min}$. The dye was dissolved in 30\% glacial acetic acid (Sigma-Aldrich), and the absorbance was measured by xMark Microplate Spectrophotometer at $590 \mathrm{~nm}$.

Motility assays. Twitching motilities were assayed on agar plates (freshly prepared LB agar plates with $1 \%$ Bacto agar were used for the twitching assay) in the presence or absence of $16 \mu \mathrm{g} / \mathrm{ml}$ hyperoside (23). An overnight culture was stabbed with a toothpick to transfer PAO1 through the agar layer (point-incubation) to the bottom of the Petri dish and plates were then incubated at $37^{\circ} \mathrm{C}$ for $48 \mathrm{~h}$. The agar was removed and attached cells were stained with $1 \%$ crystal
Table I. Primer sequences used for reverse transcriptionquantitative polymerase chain reaction.

\begin{tabular}{lll}
\hline Gene & $\begin{array}{c}\text { Primer } \\
\text { direction }\end{array}$ & \multicolumn{1}{c}{ Sequence (5'-3') } \\
\hline lasR & $\begin{array}{l}\text { Forward } \\
\text { Reverse }\end{array}$ & $\begin{array}{l}\text { CTGTGGATGCTCAAGGACTAC } \\
\text { ACCGAACTTCCGCCGAAT }\end{array}$ \\
lasI & Forward & CGTGCTCAAGTGTCAAGGA \\
& Reverse & GCGTCTGGATGTCGTTCTG \\
rhlR & Forward & CCGATGCTGATGTCCAACC \\
& Reverse & GCTACATCGTCGCCATGAG \\
rhlI & Forward & GCTACATCGTCGCCATGAG \\
& Reverse & TCTCGCCCTTGACCTTCTG \\
16S & Forward & ATCTTCGGACCTCACGCTATC \\
& Reverse & CCAACTTGCTGAACCACCTAC
\end{tabular}

violet (Sigma-Aldrich) at room temperature for $15 \mathrm{~min}$. The plates were washed gently with PBS to remove unattached cells prior to staining. The diameter of the stained zone was measured by graduated scale to assess the twitching motility.

Reverse transcription-quantitative PCR (RT-qPCR). RT-qPCR was used to detect the transcription levels of lasI, lasR, rhlI and rhlR genes in P. aeruginosa with or without $16 \mu \mathrm{g} / \mathrm{ml}$ hyperoside. The primers used to amplify these genes are listed in Table I. Total RNA was isolated from PAO1 using a FastRNA Pro Blue Kit (MP Biomedicals, Santa Ana, CA, USA). Cells were grown overnight at $37^{\circ} \mathrm{C}$ in the presence or absence of hyperoside and harvested by centrifugation at $16,100 \mathrm{xg}$ for $10 \mathrm{~min}$, and the deposit was resuspended in TRIzol (Tiangen Biotech Co., Ltd., Beijing, China). Preparation of total RNA was performed according to the manufacturer's protocol. The RNA sample was treated with 50 units of DNase I (Roche Applied Science, Penzberg, Germany) for $2 \mathrm{~h}$ at $37^{\circ} \mathrm{C}$ to remove contaminating DNA. DNaseIwas eliminated by phenol-chloroform extraction and ethanol precipitation. The pellet was resuspended in diethyl pyrocarbonate (DEPC)-treated $\mathrm{H}_{2} \mathrm{O}$. Reverse transcription was performed using the First Strand cDNA Synthesis kit (Toyobo Co., Ltd., Osaka, Japan) and qPCR using SYBR ${ }^{\circledR}$ Green Real-time PCR Master mix (Toyobo Co., Ltd.) according to the manufacturer's protocol. The reaction procedure involved two-step PCR programme: $94^{\circ} \mathrm{C}$ for $5 \mathrm{~min},\left(94^{\circ} \mathrm{C}\right.$ for $30 \mathrm{sec}, 57^{\circ} \mathrm{C}$ for $30 \mathrm{sec}$ and $72^{\circ} \mathrm{C}$ for $\left.30 \mathrm{sec}\right)$ $\mathrm{X} 40$ cycles. The $16 \mathrm{~S}$ rRNA gene was selected as the internal control to normalize the data. Relative expression of gene (RQ) was calculated by $2^{-\Delta \Delta c q}$ and percent reduction was calculated as (1-RQ) X 100 (24). Experiments were repeated independently three times.

Statistical analysis. The results are expressed as the mean \pm standard deviation and the results from at least three independent experiments were statistically analyzed by one-way analysis of variance, using SPSS 17.0 software (SPSS, 
Inc., Chicago, IL, USA). $\mathrm{P}<0.05$ was considered to represent a statistically significant difference.

\section{Results}

Effect of hyperoside on P. aeruginosa biofilm formation. Hyperoside is a flavonol glycoside with variety of biological activities, including antioxidant, anticancer, antihyperglycemic and anti-inflammatory functions (25-27). However, prior to the current study, hyperoside has demonstrated little antibacterial activity $(20,28)$. The dose-effect experiment of the present study demonstrated that $256 \mu \mathrm{g} / \mathrm{ml}$ hyperoside exhibited no inhibitory effects, and $16 \mu \mathrm{g} / \mathrm{ml}$ hyperoside had the strongest inhibitory effect on $P$. aeruginosa biofilm formation. All doses of hyperoside between 16-64 $\mu \mathrm{g} / \mathrm{ml}$ demonstrated biofilm inhibition to an extent (Fig. 1). As hyperoside concentration decreased, (from 64 to $16 \mu \mathrm{g} / \mathrm{ml}$ ) the inhibition rate of biofilm formation increased. However, the biofilm inhibition activity was not concentration dependent. A similar mode of action has been observed for another traditional medicine component, catechins (29). Although hyperoside does not exhibit antibacterial activity at experimental concentrations, higher hyperoside concentrations may have other pharmacological activities that weaken the effect of biofilm formation.

Effect of hyperoside on growth. A growth curve using $16 \mu \mathrm{g} / \mathrm{ml}$ hyperoside is presented (Fig. 2) and the negligible difference in coincident bacterial growth suggests that the biofilm inhibition effect was completely unrelated to antibacterial activity.

CLSM observation. The inhibitory effect of $16 \mu \mathrm{g} / \mathrm{ml}$ hyperoside was confirmed by CLSM micrographs of the P. aeruginosa biofilm. Figure 3 shows that the biofilm of the hyperoside group was sparse (Fig. 3B) compared with the control group (Fig. 3A) and the amount of bacteria and polysaccharide was clearly decreased.

Effect of hyperoside on adhesion. Hyperoside had a significant inhibitory effect on PAO1 cell adhesion, a process involved in initial biofilm formation ( $\mathrm{P}<0.05$; Fig. 4). The inhibition of initial adherence by hyperoside suggests that it may decrease and delay new biofilm formation.

Effect of hyperoside on motility. Twitching motility is mediated by type 4 pili (30) and this was inhibited by hyperoside (Fig. 5). Accordingly, twitching motility may be decreased by inhibiting the activity of type 4 pili. The movement of pili is the first stage of biofilm formation. As an essential step for irreversible adhesion, it can affect the morphology and structure of the biofilm. Therefore, hyperoside may inhibit the biofilm formation of $P$. aeruginosa through inhibiting its type 4 pili.

Effect of hyperoside on gene expression. P. aeruginosa has two well-studied quorum sensing (QS) systems, las and rhl. The las system is comprised of the transcriptional activator LasR and the autoinducer (AI) synthase LasI. The rhl system is comprised of the transcriptional regulatory protein RhlR and the RhlI AI synthase (31). The las and rhl systems are important for P. aeruginosa biofilm development $(32,33)$.

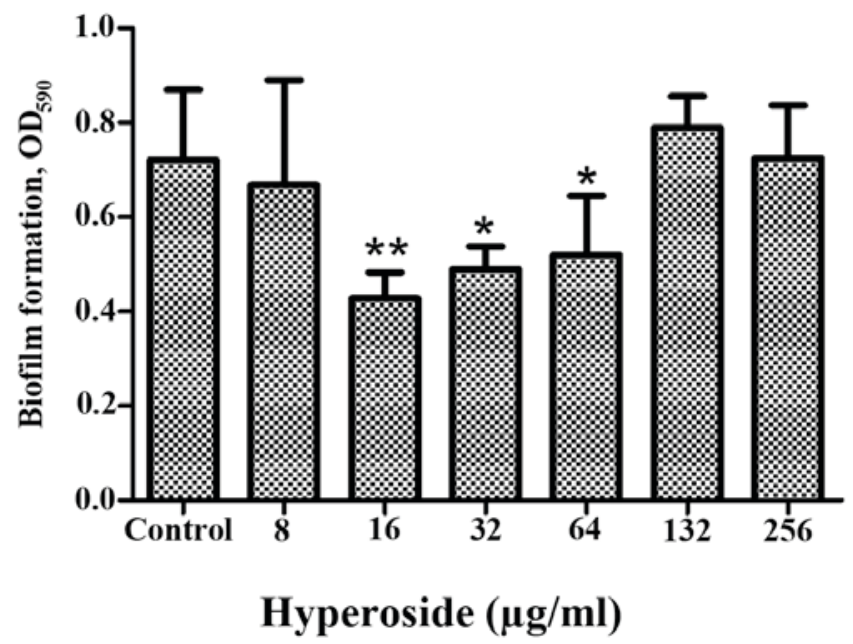

Figure 1. Effect of hyperoside on biofilm formation at different concentrations. Results are presented as the mean \pm standard deviation obtained from three independent experiments. ${ }^{*} \mathrm{P}<0.05,{ }^{* *} \mathrm{P}<0.01$ vs. the control group. $\mathrm{OD}_{590}$, optical density at $590 \mathrm{~nm}$.

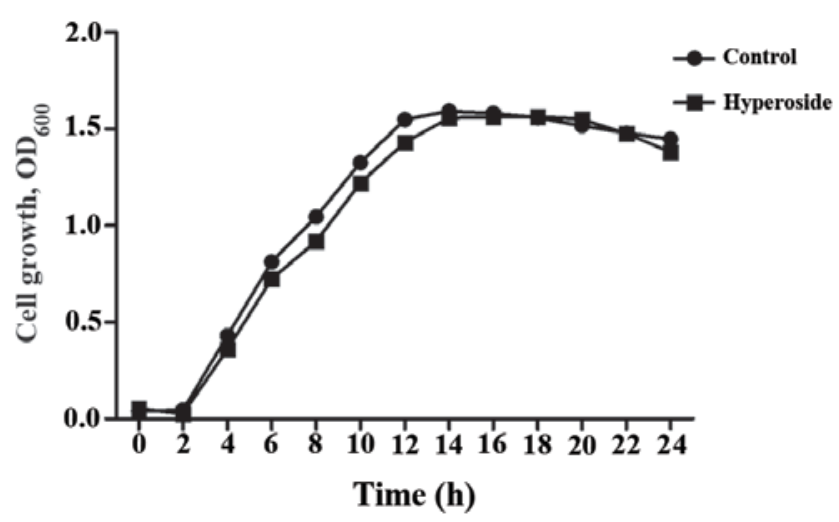

Figure 2. Growth curve of $P$. aeruginosa in the presence or absence of $16 \mu \mathrm{g} / \mathrm{ml}$ hyperoside over $24 \mathrm{~h}$. $\mathrm{OD}_{600}$, optical density at $600 \mathrm{~nm}$.

Hyperoside inhibits a multitude of factors involved in biofilm formation; therefore, it may inhibit the QS systems. RT-qPCR was used to detect lasR, lasI, rhlR and rhlI gene expression. The results from RT-qPCR indicated that hyperoside inhibited the expression of the lasR, lasI, rhlR and rhlI genes as the downregulation of transcription in these genes was significant $(\mathrm{P}<0.05$; Fig. 6). Similar effects have been observed regarding other flavonol glycosides in previous studies (34).

\section{Discussion}

Bacteria living in biofilms cause $80 \%$ of bacterial infections (35). Due to the high antibiotic resistance of biofilms, novel drugs are required to treat biofilm infections. Previous studies have shown that the formation of biofilm can be inhibited with sub-minimum inhibitory concentrations of certain antibiotics, such as imipenem and erythromycin $(36,37)$. However, the long-term use of antibiotics will give rise to drug-resistant bacteria. An increasing number of studies have found that natural products, including baicalin, allicin and garlicin, exhibit an inhibitory effect on biofilms $(38,39)$. 

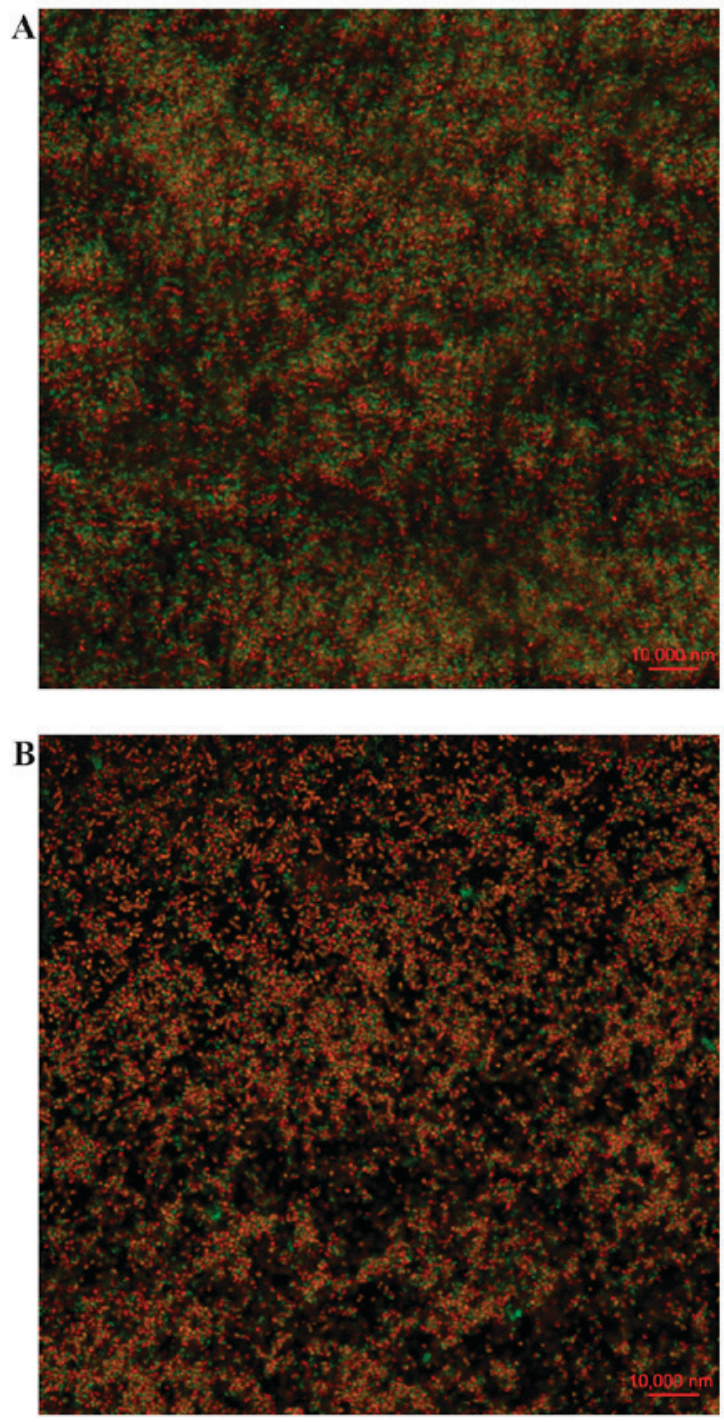

Figure 3. Confocal laser scanning microscopy showing the effect of hyperoside on P. aeruginosa biofilm formation. Concanavalin A (green) and PI (red) staining were used to generate the images. (A) CLSM image of untreated PAO1 biofilm. (B) CLSM image of PAO1 biofilm treated with $16 \mu \mathrm{g} / \mathrm{ml}$ hyperoside. CLSM, confocal laser scanning microscopy; PAO1, Pseudomonas aeruginosa PAO1.

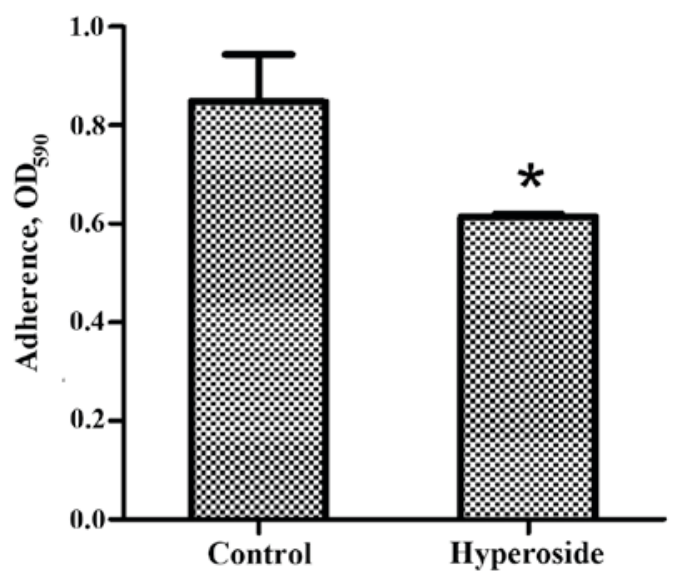

Figure 4. Adhesion ability of P. aeruginosa in the presence or absence of $16 \mu \mathrm{g} / \mathrm{ml}$ hyperoside. Results are presented as the mean \pm standard deviation as obtained from three independent experiments. " $\mathrm{P}<0.05$ vs. the control group. $\mathrm{OD}_{590}$, optical density at $590 \mathrm{~nm}$.
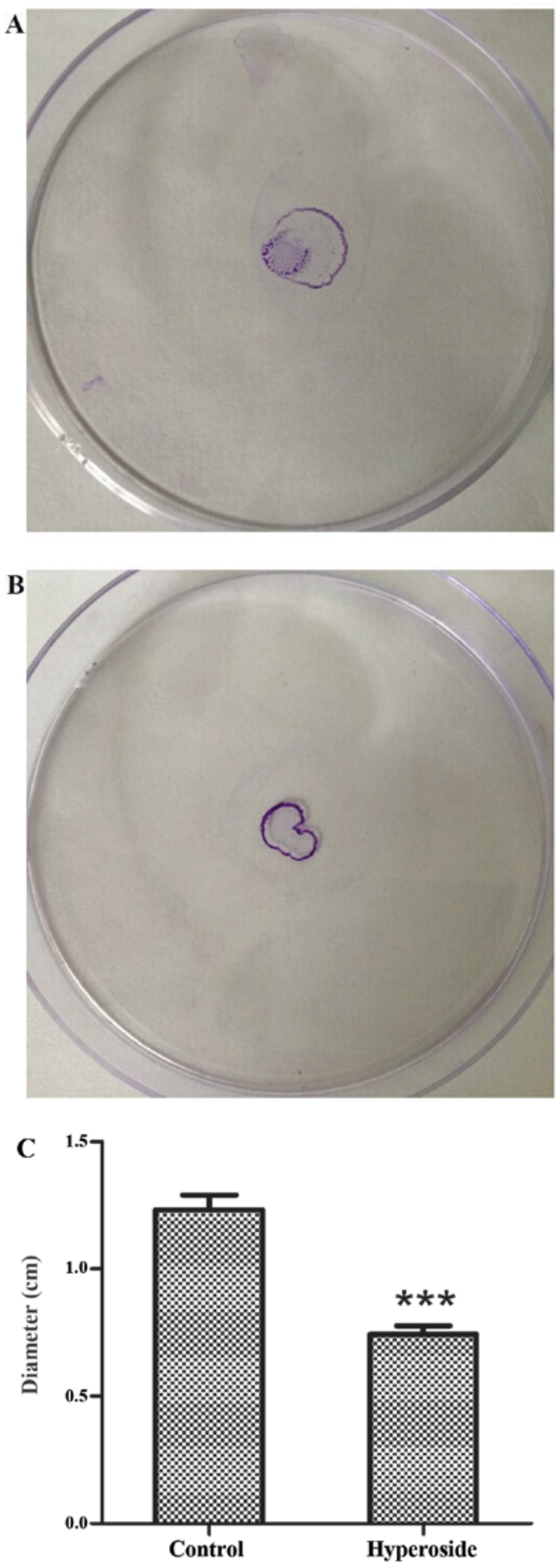

Figure 5. Twitching motility of $P$. aeruginosa standard strain PAO1. (A) Absence of hyperoside and (B) presence of $16 \mu \mathrm{g} / \mathrm{ml}$ hyperoside. (C) Graph indicating the diameter of the stained zone. Results are presented as the mean \pm standard deviation obtained from three independent experiments. ${ }^{* * *} \mathrm{P}<0.001$ vs. the control group. PAO1, Pseudomonas aeruginosa PAO1.

The use of them for biofilm inhibition has been successful and has the potential to identify novel medicines. Currently, there is no natural drug permitted for use in the clinical treatment of biofilm infection. In this study, we demonstrated that hyperoside treatment of PAO1 attenuated biofilm formation, which is related to the QS system. Twitching motility is a 


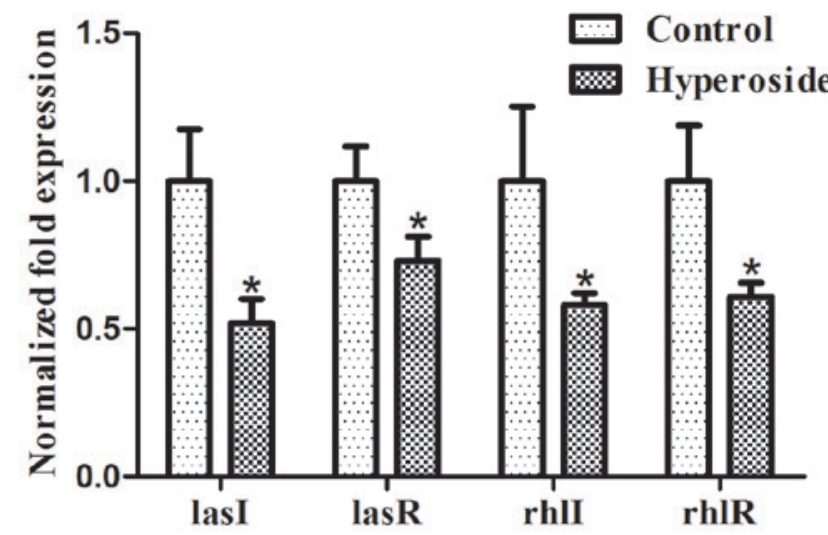

Figure 6. Comparison the transcription levels of lasI, lasR, rhlI and rhlR genes related to QS system in $P$. aeruginosa with or without $16 \mu \mathrm{g} / \mathrm{ml}$ hyperoside. Expression levels were determined by reverse transcription-quantitative polymerase chain reaction. Data are presented as the mean \pm standard deviation. The experiment was repeated three times. ${ }^{*} \mathrm{P}<0.05$ vs. control group.

type IV pili-driven movement by which bacteria can adhere and spread biofilm over a surface $(40,41)$. Type IV pili is also controlled by QS in P. aeruginosa (42). In the current study, it was found that hyperoside-treated PAO1 exhibited reduced adherence, as well as twitching motility, suggesting that hyperoside impairs the QS system to inhibit PAO1 adherence, motility and biofilm formation. Additionally, quantitative analysis of gene expression showed that hyperoside inhibited the expression of lasR, lasI, rhlR and rhlI genes involved in the QS system, which indicates that hyperoside affected $P$. aeruginosa biofilm formation by repressing the activity of las and rhl systems.

In view of its structure and function, biofilm is difficult to be removed completely by a single drug treatment. Therefore, drug combinations are often used to overcome biofilm formation, especially antibiotic-antibiotic combinations (43-45). Despite a favorable anti-biofilm effect, the combination of antibiotics can result in the generation of drug resistant bacteria, and enhance the toxicity to humans. Several studies have showed that phytochemicals can potentiate the activity of eradicating biofilm of antibiotics in combination (46-49). The combinations of antibiotic sulfamethoxazole with protocatechuic acid/ellagic acid/gallic acid and tetracycline with gallic acid, cefoperazone with allicin showed synergistic mode of interaction and were highly effective in inhibition $P$. aeruginosa biofilm under in vitro conditions (50). Vitexin has been found to potentiate the anti-biofilm activity of azithromycin and gentamicin on $P$. aeruginosa (51). As a type of nature product, whether hyperoside also has synergistic effects with antibiotics that inhibit $P$. aeruginosa biofilm formation requires further study. Although hyperoside exhibits antibacterial properties against $P$. aeruginosa in vitro, its anti-bacterial effects against $P$. aeruginosa in vivo are largely unknown. Defined clinical trials should be conducted to confirm its efficacy. Furthermore, little is known regarding the mechanism of hyperoside, and thus warrants further investigation. Overall, the effect and mechanisms of hyperoside require further assessment, including analysis using in vivo techniques.

\section{Acknowledgements}

The current study was supported by the Application Development Project of Chongqing (grant no. cstc2014yykfA 110021).

\section{References}

1. Gristina AG, Oga M, Webb LX and Hobgood CD: Adherent bacterial colonization in the pathogenesis of osteomyelitis. Science 228: 990-993, 1985.

2. Goossens H: Susceptibility of multi-drug-resistant Pseudomonas aeruginosa in intensive care units: Results from the European MYSTIC study group. Clin Microbiol Infect 9: 980-983, 2003.

3. Chatterjee M, Anju CP, Biswas L, Anil Kumar V, Gopi Mohan C and Biswas R: Antibiotic resistance in Pseudomonas aeruginosa and alternative therapeutic options. Int J Med Microbiol 306: 48-58, 2016

4. Musken M, Di Fiore S, Romling U and Häussler S: A 96-well-plate-based optical method for the quantitative and qualitative evaluation of Pseudomonas aeruginosa biofilm formation and its application to susceptibility testing. Nat Protoc 5: 1460-1469, 2010.

5. Høiby N, Krogh Johansen H, Moser C, Song Z, Ciofu O and Kharazmi A: Pseudomonas aeruginosa and the in vitro and in vivo biofilm mode of growth. Microbes Infect 3: 23-35, 2001.

6. Ceri H, Olson ME, Stremick C, Read RR, Morck D and Buret A: The Calgary Biofilm Device: New technology for rapid determination of antibiotic susceptibilities of bacterial biofilms. J Clin Microbiol 37: 1771-1776, 1999.

7. Anwar $\mathrm{H}$ and Costerton JW: Enhanced activity of combination of tobramycin and piperacillin for eradication of sessile biofilm cells of Pseudomonas aeruginosa. Antimicrob Agents Chemother 34: 1666-1671, 1990.

8. Van Delden C and Iglewski BH: Cell-to-cell signaling and Pseudomonas aeruginosa infections. Emerg Infect Dis 4: 551-560, 1998.

9. Wang Y, Wang T, Hu J, Ren C, Lei H, Hou Y and Brantner AH: Anti-biofilm activity of TanReQing, a Traditional Chinese Medicine used for the treatment of acute pneumonia. J Ethnopharmacol 134: 165-170, 2011.

10. Palombo EA: Traditional medicinal plant extracts and natural products with activity against oral bacteria: Potential application in the prevention and treatment of oral diseases. Evid Based Complement Alternat Med 2011: 680354, 2011.

11. Sayem SM, Manzo E, Ciavatta L, Tramice A, Cordone A, Zanfardino A, De Felice M and Varcamonti M: Anti-biofilm activity of an exopolysaccharide from a sponge-associated strain of Bacillus licheniformis. Microb Cell Fact 10: 74, 2011.

12. Chen X, Shang F, Meng Y,Li L, Cui Y,Zhang M, Qi K and Xue T: Ethanol extract of Sanguisorba officinalis L. inhibits biofilm formation of methicillin-resistant Staphylococcusaureus in an ica-dependent manner. J Dairy Sci 98: 8486-8491, 2015.

13. Ren S, Wu M, Guo J, Zhang W, Liu X, Sun L, Holyst R, Hou S, Fang $Y$ and Feng X: Sterilization of polydimethylsiloxane surface with Chinese herb extract: A new antibiotic mechanism of chlorogenic acid. Sci Rep 5: 10464, 2015.

14. Chen ZW, Ma CG and Xu SY: Mechanism of analgesic action of hyperin. Yao Xue Xue Bao 24: 326-330, 1989 (In Chinese).

15. Xin Q and Chen S: Advances in the study of the protection of hyperin against ischemic injuries of tissues and organs. Zhong Yao Cai 26: 213-215, 2003 (In Chinese).

16. Wang WQ, Ma CG and Xu SY: Protective effect of hyperin against myocardial ischemia and reperfusion injury. Zhongguo Yao Li Xue Bao 17: 341-344, 1996.

17. Verma N, Amresh G, Sahu PK, Mishra N, Rao ChV and Singh AP: Pharmacological evaluation of hyperin for antihyperglycemic activity and effect on lipid profile in diabetic rats. Indian J Exp Biol 51: 65-72, 2013.

18. Lee S, Park HS, Notsu Y, Ban HS, Kim YP, Ishihara K, Hirasawa N, Jung SH, Lee YS, Lim SS, et al: Effects of hyperin, isoquercitrin and quercetin on lipopolysaccharide-induced nitrite production in rat peritoneal macrophages. Phytother Res 22: 1552-1556, 2008.

19. Marčetić MD, Milenković MT, Lakušić DV and Lakušić BS: Chemical Composition and antimicrobial activity of the essential oil and methanol extract of Hypericum aegypticum subsp. webbii (Spach) N. Robson. Chem Biodivers 13: 427-436, 2016. 
20. Lee S, Shin DS, Oh KB and Shin KH: Antibacterial compounds from the leaves of Acanthopanax senticosus. Arch Pharm Res 26: 40-42, 2003.

21. Wang SS, Wang DM, Pu WJ and Li DW: Phytochemical profiles, antioxidant and antimicrobial activities of three Potentilla species. BMC Complement Altern Med 13: 321, 2013.

22. Neidig A, Yeung AT, Rosay T, Tettmann B, Strempel N, Rueger M, Lesouhaitier O and Overhage J: TypA is involved in virulence, antimicrobial resistance and biofilm formation in Pseudomonas aeruginosa. BMC Microbiol 13: 77, 2013.

23. Bala A, Kumar R and Harjai K: Inhibition of quorum sensing in Pseudomonas aeruginosa by azithromycin and its effectiveness in urinary tract infections. J Med Microbiol 60: 300-306, 2011.

24. Livak KJ and Schmittgen TD: Analysis of relative gene expression data using real-time quantitative PCR and 2(-Delta Delta C(T)) Method. Methods 25: 402-408, 2001

25. Xing HY, Liu Y, Chen JH, Sun FJ, Shi HQ and Xia PY: Hyperoside attenuates hydrogen peroxide-induced L02 cell damage via MAPK-dependent Keap-Nrf-ARE signaling pathway. Biochem Biophys Commun 410: 759-765, 2011.

26. Li W, Liu M, Xu YF, Feng Y, Che JP, Wang GC and Zheng JH: Combination of quercetin and hyperoside has anticancer effects on renal cancer cells through inhibition of oncogenic microRNA-27a. Oncol Rep 31: 117-124, 2014.

27. Ku SK, Kwak S, Kwon OJ and Bae JS: Hyperoside inhibits high-glucose-induced vascular inflammation in vitro and in vivo. Inflammation 37: 1389-1400, 2014.

28. Abedini A, Roumy V, Mahieux S, Biabiany M, Standaert-Vitse A Rivière C, Sahpaz S, Bailleul F, Neut C and Hennebelle T: Rosmarinic acid and its methyl ester as antimicrobial components of the hydromethanolic extract of Hyptis atrorubens poit (Lamiaceae). Evid Based Complement Alternat Med 2013: 604536, 2013.

29. Matsunaga T, Nakahara A, Minnatul KM, Noiri Y, Ebisu S, Kato A and Azakami H: The inhibitory effects of catechins on biofilm formation by the periodontopathogenic bacterium, Eikenella corrodens. Biosci Biotechnol Biochem 74: 2445-2450, 2010.

30. Giltner CL, van Schaik EJ, Audette GF, Kao D, Hodges RS, Hassett DJ and Irvin RT: The Pseudomonas aeruginosa type IV pilin receptor binding domain functions as an adhesin for both biotic and abiotic surfaces. Mol Microbiol 59: 1083-1096, 2006.

31. Davies DG, Parsek MR, Pearson JP, Iglewski BH, Costerton JW and Greenberg EP: The involvement of cell-to-cell signals in the development of a bacterial biofilm. Science 280: 295-298, 1998.

32. Morici LA, Carterson AJ, Wagner VE, Frisk A, Schurr JR Höner zu Bentrup K, Hassett DJ, Iglewski BH, Sauer K and Schurr MJ: Pseudomonas aeruginosa AlgR represses the $\mathrm{Rhl}$ quorum-sensing system in a biofilm-specific manner. J Bacteriol 189: 7752-7764, 2007.

33. De Kievit TR, Gillis R, Marx S, Brown C and Iglewski BH: Quorum-sensing genes in Pseudomonas aeruginosa biofilms: Their role and expression patterns. Appl Environ Microbiol 67: 1865-1873, 2001.

34. Zeng Z, Qian L, Cao L, Tan H, Huang Y, Xue X, Shen Y and Zhou S: Virtual screening for novel quorum sensing inhibitors to eradicate biofilm formation of Pseudomonas aeruginosa. Appl Microbiol Biotechnol 79: 119-126, 2008.

35. Vo GD, Brindle E and Heys J: An experimentally validated immersed boundary model of fluid-biofilm interaction. Water Sci Technol 61: 3033-3040, 2010

36. Cirioni O, Silvestri C, Ghiselli R,KamyszW, Minardi D, Castelli P, Orlando F, Kamysz E, Provinciali M, Muzzonigro G, et al: In vitro and in vivo effects of sub-MICs of pexiganan and imipenem of Pseudomonas aeruginosa adhesion and biofilm development. Infez Med 21: 287-295, 2013.
37. Zhao YL, Zhou YH, Chen JQ, Huang QY, Han Q, Liu B, Cheng GD and Li YH: Quantitative proteomic analysis of sub-MIC erythromycin inhibiting biofilm formation of S, suis in vitro. J Proteomics 116: 1-14, 2015.

38. Wang C, Cheng H, Zhang X, Xu S, Guan Y, Yu L and Yun Y: In vitro activity of baicalin against non-albicans Candida biofilms. Zhongguo Zhong Yao Za Zhi 35: 639-641, 2010 (In Chinese).

39. Saleem M, Nazir M, Ali MS, Hussain H, Lee YS, Riaz N and Jabbar A: Antimicrobial natural products: An update on future antibiotic drug candidates. Nat Prod Rep 27: 238-254, 2010.

40. Tolker-Nielsen T, Brinch UC, Ragas PC, Andersen JB, Jacobsen CS and Molin S: Development and dynamics of Pseudomonas aeruginosa sp. biofilms. J Bacteriol 182: 6482-6489, 2000.

41. Sauer K, Camper AK, Ehrlich GD, Costerton JW and Davies DG: Pseudomonas aeruginosa displays multiple phenotypes during development as a biofilm. J Bacteriol 184: 1140-1154, 2002.

42. Köhler T, Curty LK, Barja F, van Delden C and Pechére JC: Swarming of Pseudomonas aeruginosa is dependent on cell-to-cell signaling and requires flagella and pili. J Bacteriol 182: 5990-5996, 2000.

43. Olson KM, Starks CM, Williams RB, O'Neil-Johnson M, Huang Z, Ellis M, Reilly JE and Eldridge GR: Novel pentadecenyl tetrazole enhances susceptibility of methincillin-resistant Staphylococcus aureus biofilm to gentamicin. Antimicrob Agents Chemother 55: 3691-3695, 2011.

44. Pettit RK, Weber CA, Lawrence SB, Pettit GR, Kean MJ and Cage GD: In vivo activity of anprocide alone and in vitro activity in combination with conventional antibiotics against Staphylococcus aureus and Staphylococcus epidermidis biofilms. J Med Microbiol 58: 1203-1206, 2010.

45. McConeghy KW and LaPlante KL: In vitro activity of tigecycline in combination with gentamicin against biofilm-forming Staphylococcus aureus. Diagn Microbiol Infect Dis 68: 1-6, 2010.

46. Chen YQ, Zuo XJ, Zhu LN, Song ZJ, Shi HZ, Zuo P and Guo XH: In vitro effects of honeysuckle aqueous extracts alone and in combination with ceftazidime on Pseudomonas aeruginosa biofilms. Chin J Microbiol Immunol 24: 738-742, 2004.

47. Kong JL, Liu XL, Chen YQ, et al: In vitro effect of Scutellaria aqueous extracts combination with levofloxacin on Pseudomonas aeruginosa biofilm. Tianjin Med J 36: 331-333, 2008.

48. Huang XM, Huang LC, Fang ZH, et al: Synergism of Sophora flavescens ait and ciprofloxacin on Pseudomonas aeruginosa biofilms. J Shaoguan Univ 27: 60-63, 2006.

49. Zhou Q, Deng $\mathrm{CH}$, Zhang W, et al: In vitro effects of aqueous extract of Sophora in combination with ceftazidime on elimination of Pseudomonas aeruginosa biofilms. J New Chin Med 40: 98-99, 2008.

50. Jayaraman P, Sakharkar MK, Lim CS, Tang TH and Sakharkar KR: Activity and interactions of antibiotic and phytochemical combinations against Pseudomonas aeruginosa in vitro. Int J Biol Sci 6: 556-568, 2010.

51. Das MC, Sandhu P, Gupta P, Rudrapaul P, De UC, Tribedi P, Akhter Y and Bhattacharjee S: Attenuation of Pseudomonas aeruginosa biofilm formation by Vitexin: A combinatorial study with azithromycin and gentamicin. Sci Rep 6: 23337, 2016. 